ENTREVISTAS

\title{
ENTREVISTA CON FERNANDO BRONCANO
}

Profesor Broncano, agradecemos su amable disposición para participar en la revista Stoa del Instituto de Filosofía de la UV. El objetivo general de esta entrevista es conocer su opinión respecto al estado actual de los estudios CTS en Iberoamérica y, asimismo, realizar un contraste respecto a la opinión de otra importante figura filosófica dentro del contexto Iberoamericano, el profesor León Olivé, quien también, amablemente, ha decidido participar en este número de Stoa. Nos gustaría empezar preguntándole cómo caracterizaría a los estudios CTS en general y cuál sería su percepción sobre la forma en que se han desarrollado en el contexto iberoamericano.

Los estudios CTS se originan tanto en el contexto internacional como en el iberoamericano como resultado de una progresiva apertura de la filosofía de la ciencia hacia las fronteras de lo que hace años se entendían como aspectos internos del desarrollo científico. Estas fronteras eran, por un lado, la estructura interna de la ciencia como institución social y las relaciones de esa institución con el resto de las instituciones sociales y con la estructura social en general. En otro sentido los estudios CTS comenzaron a plantear el mismo lugar de la ciencia y la tecnología en el contexto económico, político y ecológico: su impacto, su contribución a la transformación y el cambio, su función polar de fuente de desarrollo pero también de fuente de riesgo y en ocasiones de dominación. Aunque hubo desde el comienzo una cierta influencia de los sociólogos que aportaban una visión relativista, tanto en el contexto internacional como en el iberoamericano el componente principal de los estudios CTS fue el aportado por los filósofos, en particular desde una perspectiva crítica, que abarcaba diversas tradiciones desde la izquierda socialista al feminismo y al ecologismo. Este impulso activista contribuyó a plantear en la ciencia discusiones que hasta esos momentos solamente habían tenido lugar en otros dominios culturales. A diferencia de un contexto internacional, sin embargo, en el contexto iberoamericano siempre hubo una voluntad de participación 
en las instituciones de evaluación y dirección de la política científica con el objeto de llevar a éstas los aspectos, valores y mirada que aportaban los estudios CTS, por ejemplo, su atención a las cuestiones de la participación democrática en las decisiones, a las cuestiones de desigualdad de género, o a las cuestiones ecológicas y de riesgo que comportan las políticas de desarrollo e innovación. De todas formas, no deberíamos concebir que los estudios CTS forman un frente homogéneo tanto en lo ideológico como en sus pretensiones prácticas. Desde su inicio se observan dos líneas no sé si divergentes, pero al menos sí con diferentes agendas respecto al futuro de los estudios CTS: de un lado una perspectiva crítica, muy similar a la de los estudios culturales, donde el horizonte de los estudios es el de poner de relieve de una forma permanente los aspectos más negativos que la ciencia y la tecnología introducen en el mundo global contemporáneo, y otra perspectiva, digamos más "ilustrada" que lo que pretende, al menos en el contexto iberoamericano, es contribuir a resolver los problemas estructurales que la investigación, el desarrollo y la innovación han tenido en nuestros países, por razones que hay que buscarlas, y en esto sí coincidiría en muchos aspectos con la anterior tradición, con ciertas características económicas, políticas y sociales de nuestra (s) cultura(s).

Desde algunos años, los estudios CTS en Iberoamérica se han intensificado con congresos, publicaciones, proyectos de investigación y en general con la interrelación de las comunidades académicas, ¿̇cree usted que puede percibirse ya un impacto social como por ejemplo en las políticas sobre investigación científica o la percepciones y actitudes sociales sobre la ciencia y al tecnología?

En la medida en que en las últimas dos décadas la investigación científica se ha convertido cada vez más en un instrumento de las políticas de desarrollo de los países, y en la medida en que se ha desarrollado un complejo sistema de financiación, evaluación y comunicación de la ciencia, muchas de las ideas circulantes en los estudios CTS han comenzado a circular e incluso materializarse en normas efectivas en todas estas instituciones. En este sentido sí puede afirmarse que los estudios CTS han producido un cambio de mirada al menos en los aspectos más institucionales de las políticas de $i+d+i$. En otros aspectos, la proyección de los estudios CTS es más discutible, sobre todo en su impacto real en las estructuras de investigación, desarrollo e innovación. Quizá por sus propias huellas y tradiciones, los estudios CTS no han permea- 
do suficientemente en las instituciones de educación científica y sobre todo no han logrado convencer suficientemente de la seriedad de sus propuestas y planteamientos a las comunidades científicas, tecnológicas y a las instituciones que realmente constituyen la armazón de la economía del conocimiento. Esta tarea sigue pendiente y no debería olvidarse, pues en otro caso su influencia no dejará de ser puramente testimonial o epidérmica.

Nos parece que un elemento persistente dentro de los estudios CTS ha sido su carácter interdisciplinar y que este carácter ha orientado no sólo los problemas teóricos sino los la función que cumple o debería cumplir la filosofía en ese contexto interdisciplinar

Por supuesto, como ocurre en otros movimientos paralelos y hasta cierto punto hermanos, como los estudios culturales, los estudios de género, y en general los estudios sociales y antropológicos, la filosofía participa al tiempo suministrando perspectivas y recibiendo una influencia auto-transformadora importante. Yo diría que los estudios CTS han contribuido a una creciente apertura de la filosofía hacia problemas que estaban anteriormente lejos de su tradición académica, muchas veces ensimismada en la discusión de aquellos problemas y conceptos que sólo recibía de su propia historia. En cualquier caso, lo que está en discusión en la propia característica "interdisciplinar" de los estudios CTS o si, por el contrario se trata más bien de una propuesta, junto con los estudios que antes mencionaba, "transdisciplinar", es decir, de una propuesta que desafía al actual aparato académico de disciplinas e instituciones educativas. Mi impresión es que hay que discutir seriamente cómo plantear la transversalidad en nuestras instituciones educativas, y cómo debemos prepararnos para una educación mucho más abierta y menos pensada desde las estructuras disciplinarias, algo que por otra parte está sucediendo con rapidez debido al proceso de transformación que implican las nuevas tecnologías.

En los últimos años han tenido un gran auge las propuestas pluralistas que intentan rescatar los saberes tradicionales y la importancia de los contextos en la conformación del conocimiento y, aún más, la relación de estos con otras formas de la racionalidad como el conocimiento científico ípodría exponernos en líneas generales cuál es su postura al respecto y cuáles son sus acuerdos y distancias respectos a los desarrollos sobre este tema que ha propuesto el profesor Olivé?

León Olivé ha planteado con rigor y radicalidad las consecuencias que debe tener tanto para las políticas científicas como sobre todo para la concepción 
filosófica implícita en ellas el hecho de la composición plural de nuestras sociedades. Una pluralidad que es identitaria en lo cultural pero también una pluralidad que significa un problema pendiente para las constituciones y la estructura económica de nuestros países. Pluralismo y contextualismo forman parte del programa de León Olivé con el que coincido en casi su totalidad. Mis diferencias, si las hay, tienen que ver con las preguntas que me hago sobre la agenda futura. El pluralismo es ya un punto de partida irrenunciable, pero no queda claro que sea el punto de llegada. Podemos organizar nuestras sociedades de modo que acojan las diferencias culturales, étnicas, de género, etc., y podemos hacerlo de manera que las políticas de igualdad no interfieran con las exigencias de respeto a las diferencias. Pero tarde o pronto nos esperan problemas que tienen que ver, por una parte con los viejos problemas de bienes públicos que están por encima de las diferencias, pero sobre todo con los cruces de identidades que cada vez forman más parte del paisaje cultural de nuestro tiempo: grupos que forman parte de varias identidades y que tienen agendas incómodas o conflictivas para todas ellas. Estoy pensando, por ejemplo, en la riquísima fuente de experiencias y creatividad para pensar que significan, por ejemplo, el México fronterizo o el México cosmopolita, donde los cruces son la regla más que la excepción. La pluralidad, aquí, se da por supuesta, pero no impide la efervescencia de políticas radicalmente innovadoras en lo cultural y posiblemente también en lo social y político.

Dentro de los estudios CTS nosotros pensamos la ciencia como una estructura abierta (por ejemplo, su dimensión histórica y cultural), que constituye sólo uno de los nodos de la racionalidad en general, el cual no tiene límites precisos y definidos respeto a otras formas de experiencia. ¿Cree usted que sea una manera adecuada de entender la empresa científica en el contexto de los estudios CTS?

Estoy radical y militantemente de acuerdo con esta perspectiva. Si en algo podemos contribuir de forma positiva al futuro de la ciencia y la tecnología en nuestras sociedades es precisamente a ayudar a las mismas comunidades de investigación a que se vean a sí mismas bajo categorías de contingencia y situacionalidad que las alejen del autoritarismo e imperialismo que muchas veces han ejercido sobre la cultura. A veces los filósofos hemos cooperado a enquistar una situación social de división de culturas afirmando la existencia de dos racionalidades. Creo más bien que hay que subrayar los aspectos histórico, 
contingente, fenomenológico, situado de la racionalidad científica y tecnológica y los aspectos de capacidad estructurante y de elaboración de la experiencia humana que tienen las racionalidades que se ejercen en otros aspectos de la cultura como las humanidades, el arte y la literatura o el pensamiento social. Creo que podemos ayudar a combatir por una parte el dogmatismo de un lado y el resentimiento del otro.

Como mencionábamos al principio, uno de los objetivos de esta entrevista es contrastar su visión de los estudios CTS con la de otro reconocido estudioso y figura fundamental de los estudios CTS en Iberoamérica, el profesor león Olivé; al respecto, quisiéramos saber cuál es su valoración sobre la propuesta del profesor Olivé, cuáles son, en general, los ámbitos de acuerdo y de desacuerdo.

Me resulta muy difícil responder a esta pregunta, porque siempre he tenido la impresión y la experiencia de estar del lado de León Olivé en muchas de las discusiones y controversias en las que nos hemos embarcado en la comunidad iberoamericana en los últimos años. Me une a él la combinación de la tradición crítica con una atención y cercanía al funcionamiento real de las instituciones de investigación y educativas, también la combinación de búsqueda de una seriedad filosófica que no impida los compromisos prácticos. Su reivindicación de los valores en la ciencia es algo que me une también en una misma empresa. Quizá yo insista más en la importancia que tiene la tecnología y la cultura material en la ciencia contemporánea. Pero no creo que dé lugar a una controversia sino más bien a una propuesta complementaria. Me acerca mucho también a León Olivé la perspectiva amplia que siempre ha tenido sobre las agendas filosóficas de nuestras comunidades. Cuando el tiempo pase, observaremos la influencia estructural que ha tenido León Olivé en la conformación de la filosofía que piensa en los ámbitos latinoamericanos, una influencia que ha sido a la vez modernizadora y respetuosa con la tradición propia.

¿Le parece a usted que haya un suelo común en el sentido filosófico, cultural y político entre las propuestas de Olivé y la suya propia y en sí la de todos los especialistas en Iberoamérica?

Decididamente sí. Me parece además que la agenda que León Olivé ha ayudado a instaurar en nuestros países tiene que ver con la creación y desarrollo de una tradición común que no se oponga por sistema, sino que complemente 
y mejore, a la actual importancia que tiene la tradición anglosajona. Pero como ocurre en filosofía, y también en CTS, debe ser la controversia, la apertura de debates y la creación de nuevas perspectivas lo que nos configure como una tradición viva. Precisamente en este aspecto el pluralismo de León Olivé es posiblemente la mejor opción en vez de muchas otras propuestas que siempre implican una toma de partido unilateral que cierra más que abre las controversias.

Ahora que hemos mencionado la comunidad iberoamericana para los estudios CTS, con un propósito comparativo ¿̇cuáles cree usted que sean los problemas específicos que deben afrontar respectivamente España y México desde la perspectiva CTS?

Hay muchos problemas de contenido común que tienen que ver con el mutuo carácter poliédrico, multicultural y conflictivo de nuestras dos sociedades, que tienen que ver también con nuestra situación de fronteras con zonas culturales, económicas y sociales de una dimensión enorme en su influencia. El hacer de esta experiencia el motor de reflexión es parte de lo que nos une en un programa de trabajo apasionante. Tenemos también el problema de tener una tradición científica no tan consolidada más que en algunas zonas marginales. Es como tratar a un paciente que se encuentra en un estado de suma debilidad y al que hay que mantener vivo al tiempo que se interviene de manera radical sobre sus males. Sin embargo tenemos la suerte invalorable de formar parte de dos comunidades extraordinariamente creativas, atentas a lo que está ocurriendo, orgullosas de su tradición al tiempo que autocríticas con respecto a ella. Tenemos un humus tan extraordinario para hacer crecer el pensamiento que lo increíblemente tonto es que desaprovechemos esta riqueza. Tenemos pendiente, y yo creo que es parte de nuestra obligación, el contribuir a integrar de manera institucional nuestras comunidades de investigación y de educación.

Insistiendo en la pregunta, ¿̇ómo considera las políticas científicas en España y en México y su relación con los problemas que se suelen discutir desde la perspectiva crítica de los estudios CTS, como las dificultades que plantea la visión tradicional de la ciencia?

Me parece que tenemos pendiente por resolver la contradicción de la que hablaba anteriormente: cómo contribuir a desarrollar una perspectiva crítica sobre la ciencia y la tecnología que, sin embargo, no tenga como subproducto no querido el hacerlas más débiles de lo que ya son con respecto al contex- 
to mundial. Hacer confiables a los formados en las tradiciones CTS ante las comunidades científicas es nuestro principal problema por el momento.

Profesor Broncano, le agradecemos su tiempo y su amabilidad para concedernos esta entrevista. Y reiteramos nuestro reconocimiento por sus esfuerzos y propuesta los cuales consideramos elementos claves en el impacto social de los estudios CTS en Iberoamérica.

Ana Luisa Ponce MiotTi 